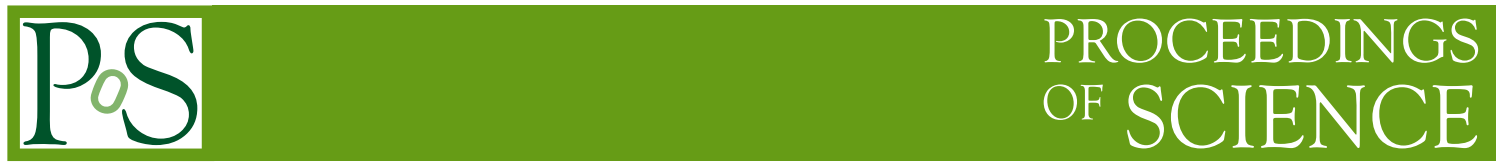

\title{
Recent developments in Multi-Channel Algebraic Scattering calculations
}

\author{
L. Canton*1, P. R. Fraser ${ }^{1}$, K. Amos ${ }^{2,3}$, S. Karataglidis ${ }^{3}$, J. P. Svenne ${ }^{4}$, \\ D. van der Knijff ${ }^{2}$ \\ ${ }^{1}$ Istituto Nazionale di Fisica Nucleare, Sezione di Padova, I-35131, Italy \\ ${ }^{2}$ School of Physics, University of Melbourne, Victoria 3010, Australia \\ ${ }^{3}$ Department of Physics, University of Johannesburg, South Africa \\ ${ }^{4}$ Department of Physics and Astronomy, University of Manitoba, Winnipeg, Manitoba, Canada
}

\begin{abstract}
A multi-channel algebraic scattering (MCAS) method has been developed to obtain spectra of a number of light-mass nuclei, which are treated as two-cluster systems. The method has been applied to a variety of cases that could be described as nucleon-core systems, but also alpha-core systems have been treated. The MCAS method gives sub-threshold/bound-state spectra, describes resonances, from the very narrow to the broad ones, and provides various scattering/reaction observables. Collective models have been used to specify the interactions between the projectile and low-lying states of the target nucleus that form the compound. In this contribution we discuss in particular some resonance features in the scattering of the $\mathrm{p}+{ }^{14} \mathrm{O}$ and $\mathrm{p}+{ }^{6} \mathrm{He}$ systems.
\end{abstract}

X Latin American Symposium on Nuclear Physics and Applications (X LASNPA),

1-6 December 2013

Montevideo, Uruguay

\footnotetext{
* Speaker.
} 


\section{Introduction}

The multi-channel algebraic scattering (MCAS) method solves the coupled-channel LippmannSchwinger (LS) equations describing a two-cluster system, in both bound-state (sub-thresholds) and scattering regimes. Most applications, to date, have involved a system of a nucleon and an even-mass nucleus $[1,2,3,4]$, but applications are in progress for alpha-core systems, as well as for core nuclei that are not of even mass.

Collective models have been used to specify the interactions between the projectile and lowlying states of the target-nucleus that form the compound. The solution of the LS equations is gained by an expansion in terms of coupled-channel sturmian functions, defined using the specified matrix of potentials. After truncation, the sturmian representation leads to a finite-rank expansion of the potential, and to an algebraic solution of the scattering problem. In defining the sturmians in the selected set, we consider in addition orthogonalising pseudo-potentials (OPPs) [5]. The OPP technique was developed in studies of cluster physics $[6,7]$ as a variant of the Orthogonality Condition Model (OCM) of Saito [8]. It accounts for the effects of Pauli blocking in the relative motion of two clusters comprised of fermion constituents. Investigations into the use of the OPP for partially forbidden orbits have shown promising results [9]. A detailed presentation of this concept is given also in [2].

No reaction channel other than that of nucleon emission has been included explicitly in the process, hence the resonance widths found are those for nucleon emission only. In recent works [10, $11,12,13]$, we have extended the method to include the possibility that the target excited states may themselves be unstable resonant states. This capability gives a more realistic description of the coupled-channel problem and leads, generally, to widths of resonant states in the compound system that include effectively other decay channels, beyond the single nucleon emission decay.

In 2006, we published [9] results of MCAS calculations on the mirror system ${ }^{15} \mathrm{C}$ (as $\mathrm{n}+{ }^{14} \mathrm{C}$ ) and ${ }^{15} \mathrm{~F}$ (as $\mathrm{p}+{ }^{14} \mathrm{O}$ ). For the ${ }^{15} \mathrm{C}$ calculations, parameters for the neutron-nucleus potentials were chosen to give a good description of the low-excitation energy levels. A Coulomb potential was then added to that nuclear potential to describe properties of the $\mathrm{p}+{ }^{14} \mathrm{O}$ cluster. With the reproduction the two known positive-parity resonances in ${ }^{15} \mathrm{~F}$, narrow negative-parity resonances above those were predicted by the MCAS calculations. In 2009, experimental results were published by Mukha, et al. [14, 15], which showed the existence of such higher-energy narrow resonances in the energy region we considered. This showed a predictive character in our study.

In this paper, we present also some new, preliminary, results for the cross section of $\mathrm{p}+{ }^{6} \mathrm{He}$ scattering and compare with experimental results that have been presented at this Conference [16].

\section{Scattering of $p+{ }^{14} \mathrm{O}$}

The low-excitation spectrum of ${ }^{14} \mathrm{O}$ has a $0^{+} ; 1$ ground state followed by a band starting $0.545 \mathrm{MeV}$ above the proton ${ }^{13} \mathrm{~N}$ threshold and consisting of a $1^{-} ; 1$ state at $5.173 \mathrm{MeV}$, and the known proton-unstable resonances with spin-parities of $0^{+} ; 1$ at $5.920 \mathrm{MeV}$, of $3^{-} ; 1$ at $6.272 \mathrm{MeV}$, and of $2^{+} ; 1$ at $6.590 \mathrm{MeV}$.

Of the low energy spectrum, the $0_{2}^{+}$and $2^{+}$were selected for coupling, as in the mirror $n+{ }^{14} \mathrm{C}$ system they generated the negative low-energy parity states in ${ }^{15} \mathrm{C}$ [9]. In this calculation, both 
Table 1: Low-lying levels of ${ }^{15} \mathrm{~F}$. Only the lowest two states were known esperimentally [17] at the time of the calculation [9]. The remaining three have been observed in Ref. [14]

\begin{tabular}{|c|cc|}
\hline \hline$J^{\pi}$ & Theory $E,\left(\frac{1}{2} \Gamma\right)$ & Experiment $E,\left(\frac{1}{2} \Gamma\right)$ \\
\hline$\frac{1}{2}^{+}$ & $1.31(0.8)$ & $1.47(1.00)$ \\
$\frac{5}{2}^{+}$ & $2.78(0.3)$ & $2.77(0.24)$ \\
\hline$\frac{1}{2}^{-}$ & $5.49(0.005)$ & $4.9(<0.2)$ \\
$\frac{5}{2}^{-}$ & $6.88(0.01)$ & $6.4(<0.2)$ \\
$\frac{3}{2}^{-}$ & $7.25(0.04)$ & \\
$\frac{1}{2}^{+}$ & $7.21(1.2)$ & \\
$\frac{5}{2}^{+}$ & $7.75(0.4)$ & $7.8(0.4) ?$ \\
$\frac{3}{2}^{+}$ & $7.99(3.6)$ & $?$ \\
\hline \hline
\end{tabular}

are taken as zero-width (ignoring their known proton-decay widths) while in a more recent calculation [11], we took into account the upper limits of the known width of the two excited states $0_{2}^{+}$ and $2^{+}$.

The coupled-channel nuclear interaction was generated assuming the collective model with rotor character for the structure. Parameter values were chosen to be those used in Ref. [9]. The compound system, ${ }^{15} \mathrm{~F}$, is particle unstable and, until recently, only its ground and first excited resonance states were known. We used known properties of ${ }^{15} \mathrm{C}$ to predict [9] new states in ${ }^{15} \mathrm{~F}$, in particular three narrow resonances of negative parities $\frac{1}{2}^{-}, \frac{3}{2}^{+}$, and $\frac{5}{2}^{+}$in the range 5-8 $\mathrm{MeV}$ of excitation.

Three years later, definite spin-parity assignments have been made for the lowest three experimentally known states while the fourth and fifth given in Ref. [14], tentatively are $\frac{3}{2}^{-}$or $\frac{5}{2}^{-}$ for the $6.4 \mathrm{MeV}$ state and $\frac{3}{2}^{+}$or $\frac{5}{2}^{+}$for the $7.8 \mathrm{MeV}$ state. These resonance state centroids are compared with the spectra predicted from our MCAS calculations in Fig. 1. The measured states pair up quite reasonably with the calculated ones. Further comparison between the predictions and the experiments is made in Table 1.

It should be noted that the parameters that produced this spectrum were fitted only to the observed $\frac{1}{2}^{+}$and $\frac{5}{2}^{+}$states, before the results of Ref. [14] were published.

\section{Scattering of $p+{ }^{6} \mathrm{He}$}

In this section, we reconsider the low-excitation spectra for ${ }^{7} \mathrm{Li}$. The division of bound and resonant states along with the calculated resonant state widths relate to the specific reaction studied. The threshold energies of diverse particle emissions relevant to ${ }^{7} \mathrm{Li}$ (and the mirror ${ }^{7} \mathrm{Be}$ ) are listed in Table 2.

Energy levels of ${ }^{7} \mathrm{Li}$ found using MCAS for different configurations are listed in Table 3. All of the energies are in $\mathrm{MeV}$. (The widths in parenthesis are in $\mathrm{keV}$ ). The experimental values [18] are compared with results calculated for the $p+{ }^{6} \mathrm{He}$ system.

Clearly the threshold energies for the proton break-up of ${ }^{7} \mathrm{Li}$ lie above the zero-energy range in the MCAS evaluations, whence all negative-energy levels found are sub-threshold and thus have no 


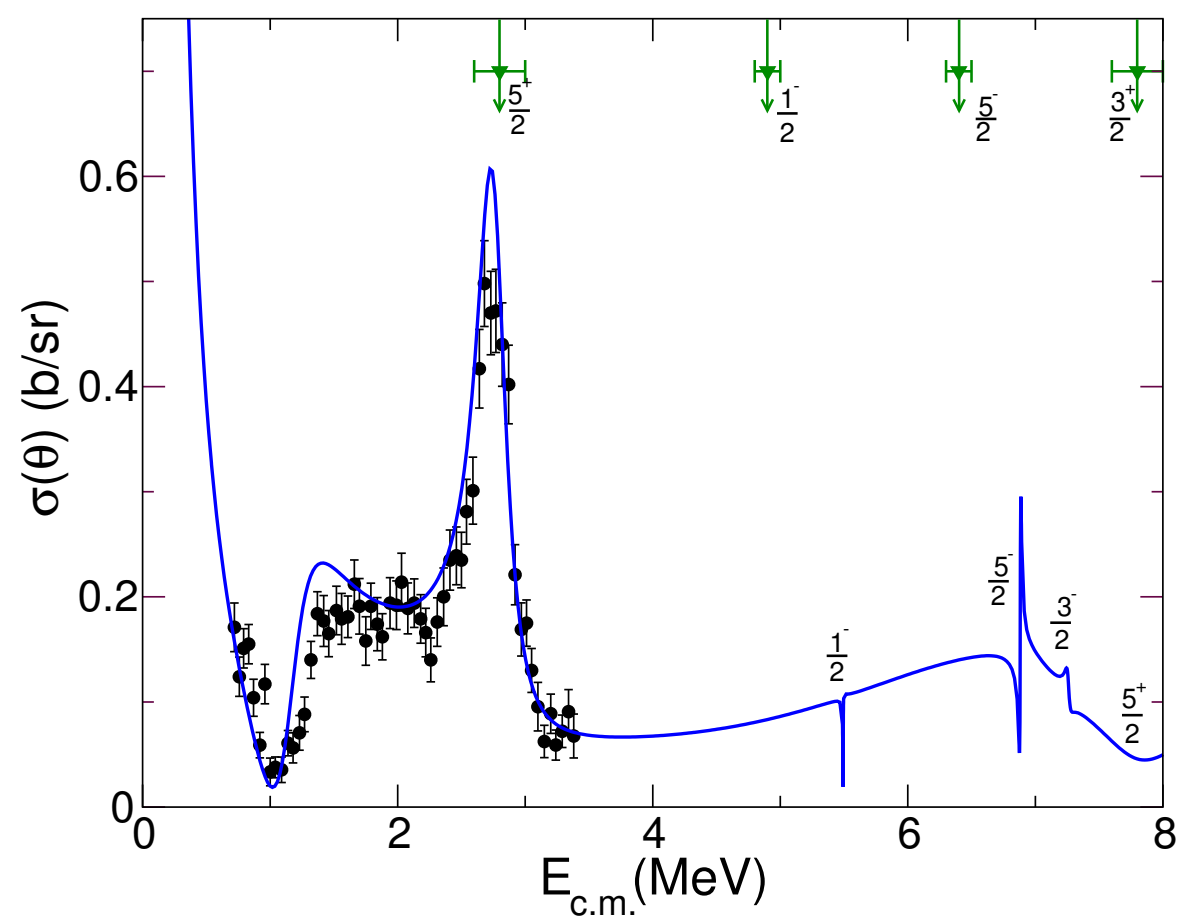

Figure 1: MCAS calculation [9] of the differential cross section at $\theta=147^{\circ}$, c.m. The horizontal bars on the down arrows at the top indicate the widths (FWHM) of the experimental ${ }^{15} \mathrm{~F}$ spectrum $[14,15]$.

Table 2: Threshold energies of reactions whose compound systems are ${ }^{7} \mathrm{Li}$ and ${ }^{7} \mathrm{Be}$.

\begin{tabular}{cccc} 
Reaction & ${ }^{7} \mathrm{Li}(\mathrm{MeV})$ & Reaction & ${ }^{7} \mathrm{Be}(\mathrm{MeV})$ \\
\hline$\alpha+{ }^{3} \mathrm{H}$ & 2.47 & $\alpha+{ }^{3} \mathrm{He}$ & 1.59 \\
${ }^{6} \mathrm{Li}+n$ & 7.25 & ${ }^{6} \mathrm{Li}+p$ & 5.61 \\
${ }^{5} \mathrm{He}+d$ & 9.52 & ${ }^{5} \mathrm{Li}+d$ & 8.77 \\
${ }^{6} \mathrm{He}+p$ & 9.98 & ${ }^{6} \mathrm{Be}+n$ & 10.6
\end{tabular}

(proton emission) width. Experimentally, the widths include alpha emissions, and therefore have decay widths also at negative energies (reported with square parenthesis in Table 3).

The decay into the $\alpha-{ }^{3} \mathrm{H}$ and $\alpha-{ }^{3} \mathrm{He}$ channels have been evaluated in Ref. [19]. Those MCAS calculations resulted in the ground and first excited states of the mass-7 nuclei being sub-threshold while the next two were resonances. Those two resonance states lie below any other emission threshold and the MCAS widths agree well with the experimental ones. In [19], we also studied ${ }^{7} \mathrm{He}$ (as $n+{ }^{6} \mathrm{He}$ ) and the mirror system, ${ }^{7} \mathrm{~B}$ (as $p+{ }^{6} \mathrm{Be}$ ). These compound systems lie beyond the relevant nucleon drip lines, with the ground state of ${ }^{7} \mathrm{He}$ being $0.445 \mathrm{MeV}$ above the neutron breakup and with a width of $0.15 \mathrm{MeV}$. Using MCAS, the ground state resonance of ${ }^{7} \mathrm{He}$ was centred at $0.43 \mathrm{MeV}$ with a width of $0.1 \mathrm{MeV}$. On the other hand, the ground state resonance of ${ }^{7} \mathrm{~B}$ not only lies $2.21 \mathrm{MeV}$ above the proton emission threshold, but it also lies $3.58 \mathrm{MeV}$ above the $\alpha+3 p$ threshold. The measured width of ${ }^{7} \mathrm{~B}(1.4 \mathrm{MeV})$ encompasses both break-up processes and so the MCAS result for just the $p+{ }^{6} \mathrm{Be}$ reaction of $0.19 \mathrm{MeV}$ need be understood in that context. 
Table 3: Experimental data and theoretical results for ${ }^{7} \mathrm{Li}$ (Energies are in $\mathrm{MeV}$, widths are in $\mathrm{keV}$ ). All energies are defined with thresholds, $p+{ }^{6} \mathrm{He}=9.975 \mathrm{MeV}$ with respect to ${ }^{7} \mathrm{Li}$ ground state. See text for explanation of the labels "orig", "II", and "III".

\begin{tabular}{|c|c|c|c|c|}
\hline$J^{\pi}$ & \multicolumn{2}{|c|}{${ }^{7} \mathrm{Li}$} & \multicolumn{2}{|c|}{${ }^{7} \mathrm{Li}$} \\
\hline & Exp. & Theory (orig) & Theory (II) & Theory (III) \\
\hline$\frac{3}{2}^{-}$ & -9.975 & -9.975 & -9.9200 & -9.9200 \\
\hline$\frac{1}{2}^{-}$ & -9.497 & -9.497 & -9.3165 & -9.3165 \\
\hline$\frac{7}{2}^{-}$ & $-5.323[69]$ & -5.323 & -5.2431 & -5.2431 \\
\hline$\frac{5}{2}^{-}$ & $-3.371[918]$ & -3.371 & -3.3661 & -3.3661 \\
\hline$\frac{5}{2}^{-}$ & $-2.251[80]$ & -0.321 & $0.1248(7 x E-6)$ & $0.1250(65)$ \\
\hline$\frac{3}{2}^{-}$ & -1.225 [4712] & -2.244 & -2.228 & -2.228 \\
\hline$\frac{1}{2}^{-}$ & $-0.885[2752]$ & -0.885 & -0.5283 & -0.5283 \\
\hline$\frac{7}{2}^{-}$ & -0.405 [437] & -0.405 & $0.1869(3 \times E-4)$ & $0.1871(85)$ \\
\hline$\frac{3}{2}^{-}$ & $1.265(260)$ & $0.704(56)$ & $1.3034(140)$ & $1.3044(223)$ \\
\hline$\frac{1}{2}^{-}$ & & $1.796(1570)$ & $2.1151(1628)$ & $2.1301(1714)$ \\
\hline$\frac{3}{2}^{-}$ & $3.7(800) ?^{a}$ & 2.981 (990) & 3.1053 (839) & 3.1158 (891) \\
\hline$\frac{5}{2}^{-}$ & $4.7(700) ?^{a}$ & $3.046(750)$ & 3.3260 (876) & 3.3352 (942) \\
\hline$\frac{5}{2}^{-}$ & & $5.964(230)$ & 6.0137 (254) & $6.0361(318)$ \\
\hline$\frac{7}{2}^{-}$ & & $6.76(2240)$ & $6.9619(2420)$ & $6.9642(2072)$ \\
\hline
\end{tabular}

${ }^{a}$ For these states spin and parity are unknown [18].

${ }^{b}$ Spin-parity of this state has been assigned as $\frac{1}{2}^{-}[18]$.

We stress that the MCAS model defines bound and resonance states of the cluster compound system according to the specific cluster threshold. Widths found for resonances then are for the specific break-up. When the threshold of the particular cluster is the lowest of all possible ones, then for energies below the very next cluster threshold, the evaluated resonance widths are the total ones. When that is not the case, the evaluated widths are partial ones.

In Fig. 2 we compare the results of MCAS calculations for low-energy scattering of proton${ }^{6} \mathrm{He}$, with results from experiments performed at the University of São Paolo. The experiment has been performed utilizing the double solenoid system Radioactive Ion Beams in Brasil (RIBRAS) $[16,20]$. Cross sections have been obtained by impinging a pure ${ }^{6} \mathrm{He}$ secondary beam on a thick $\mathrm{CH} 2$ target, to measure the ${ }^{6} \mathrm{He}+\mathrm{p}$ excitation function. Data have been recently published in Ref. [21].

In this contribution we show only the preliminary results for the cross section at $180^{\circ}$ (c.m.), while a more extended MCAS study of that resonance is in progress.

The two calculated cross sections shown as lines in Fig. 2 were obtained with the same model described in Ref. [19]. That original calculation (reported also in second column of Table 3) perfectly describes 6 subthreshold states (out of 8 in total) but offsets the centroid of the measured $1.265 \mathrm{MeV} \frac{3}{2}^{-}$resonance by about $0.5 \mathrm{MeV}$ (lower). To correct the centroid of the resonance we have included phenomenologically a small repulsion (i.e. hindrance) of $0.85 \mathrm{MeV}$ in the OPP strengths of the p-waves, specifically for the second $2^{+}$state of ${ }^{6} \mathrm{He}$ at $5.6 \mathrm{MeV}$. The calculated 

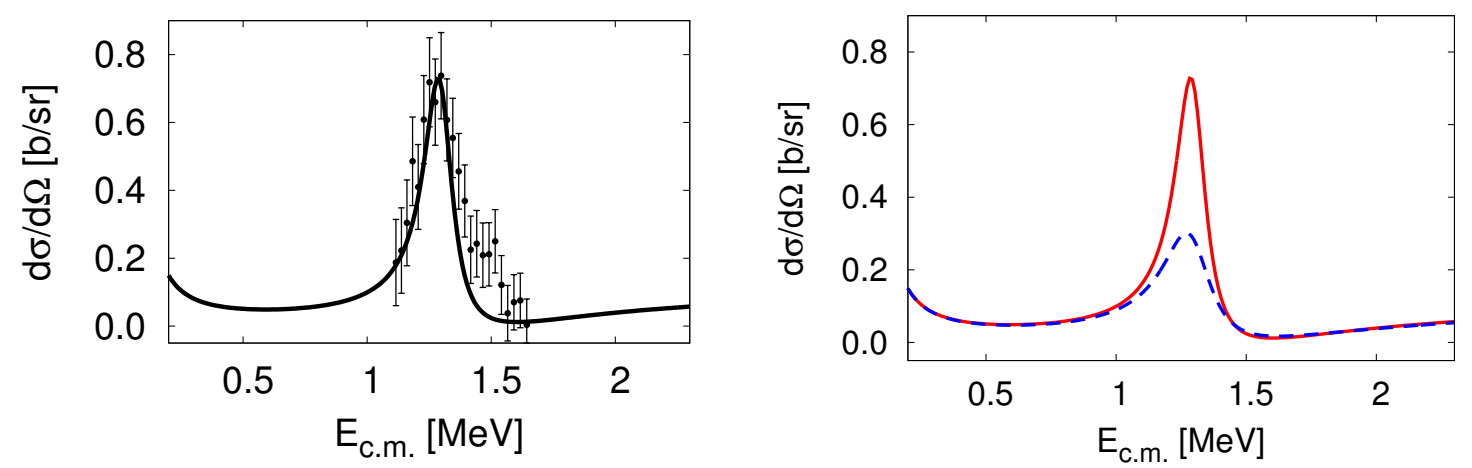

Figure 2: The angular cross section for $\mathrm{p}+{ }^{6} \mathrm{He}$ elastic scattering at $180^{\circ}$ in c.m. In the left panel we compare our calculation with measurements taken at RIBRAS [16, 21]. The calculation refers to "Theory(II)" in Table 3. The same calculation is reported in the right panel as the solid red line. It is compared with another calculation (denoted "Theory(III)" in Table 3 and represented with the dashed blue line) where we consider the decay width of the $2_{2}^{+}$level of ${ }^{6} \mathrm{He}$ in the coupled-channel formalism.

spectrum is given in the third column of Table 3, labeled as "Theory(II)". The $\frac{3}{2}^{-} 1.265$ resonance is much better reproduced in this case, while the overall spectrum is still satisfactory. We show, in the left panel of Fig. 2, the cross section obtained with this set of calculations. That compares well with the observed data, although the calculated width might be underestimated for the reasons above mentioned. To overcome this problem, we have considered, in addition, a decay width in the second $2^{+}$state of ${ }^{6} \mathrm{He}$ at $5.6 \mathrm{MeV}$. According to Ref. [18], this state has a large decay width into $2 \mathrm{n}+\alpha$. We take into account this effect by adding a width of $120 \mathrm{keV}$ to this $2_{2}^{+}$state, when it is embedded in the compound ${ }^{7} \mathrm{Li}$. The methodology is explained in Refs. $[10,11,12,13]$. The spectrum of ${ }^{7} \mathrm{Li}$, with the addition of this new feature, is given by the fourth column in Table 3 . The resulting cross sections were reported in the right panel of Fig. 2 by the blue (dashed) line. In this case, the width of the observed resonance is more correctly described, but then one should consider a possible need for normalization of the cross-section data.

\section{References}

[1] K. Amos, L. Canton, G. Pisent, J. P. Svenne and D. van der Knijff, Nucl. Phys. A 728, 65 (2003).

[2] K. Amos, L. Canton, P. R. Fraser, S. Karataglidis, J. P. Svenne and D. van der Knijff, Nucl. Phys. A 912, 7 (2013).

[3] K. Amos, L. Canton, P. R. Fraser, S. Karataglidis, J. P. Svenne and D. van der Knijff, Nucl. Phys. A 879, 132 (2012).

[4] S. Karataglidis, K. Amos, P. Fraser, L. Canton and J. P. Svenne, Nucl. Phys. A 813, 235 (2008).

[5] L. Canton, G. Pisent, J. P. Svenne, D. van der Knijff, K. Amos and S. Karataglidis, Phys. Rev. Lett. 94, 122503 (2005).

[6] V. Krasnopolsky and V. Kukulin, Soviet J. Nucl. Phys. 20, 883 (1974).

[7] V. Kukulin and V. Pomerantsev, Ann. of Phys. 111, 330 (1978). 
[8] S. Saito, Prog. Theor. Phys. 41, 705 (1969).

[9] L. Canton, G. Pisent, J. P. Svenne, K. Amos and S. Karataglidis, Phys. Rev. Lett. 96, 072502 (2006).

[10] P. R. Fraser, K. Amos, L. Canton, G. Pisent, S. Karataglidis, J. P. Svenne, and D. van der Knijff, Phys. Rev. Lett. 101, 242501 (2008).

[11] P. R. Fraser, K. Amos, L. Canton, S. Karataglidis, J. P. Svenne, and D. van der Knijff, Rev. Mex. Fis. 57, 20 (2011).

[12] L. Canton, P. R. Fraser, J. P. Svenne, K. Amos, S. Karataglidis, and D. van der Knijff, Phys. Rev. C 83, 047603 (2011).

[13] P.R. Fraser, L. Canton, R. Fossion, K. Amos, S. Karataglidis, J.P. Svenne, and D. van der Kniff, EPJ Web of Conferences 63, 02010 (2013)

[14] I. Mukha et al., Phys. Rev. C 79, 061301 (R) (2009).

[15] I. Mukha et al., Phys. Rev. C 82, 054315 (2010).

[16] R. Lichtenthäler et al., see Proceedings of the present Conference (2014), and private communication.

[17] V. Z. Goldberg, et al., Phys. Rev. C 69, 031302(R) (2004); F. Q. Guo, et al., Phys. Rev. C 72, 034312 (2005).

[18] D. R. Tilley, C. M. Cheves, J. L. Godwin, G. M. Hale, H. M. Hoffman, J. H. Kelley, C. G. Sheu, and H. R. Weller, Nucl. Phys. A708, 3 (2002).

[19] L. Canton, G. Pisent, K. Amos, S. Karataglidis, J. P. Svenne, and D. van der Knijff, Phys. Rev. C 74, 064605 (2006).

[20] R. Lichtenthäler et al., Eur. Phys. J. A 25733 (2005); R. Lichtenthäler, et al., AIP Conf. Proc. 1529, 197 (2013).

[21] R. Pampa Condori et al., Proceedings of the INPC-2013 Conference. EPJ Web of Conferences 66, 03054 (2014). 